\title{
Resection of Nasal Glial Heterotopia Using a Nasal Subunit Approach
}

\author{
Michael T. Friel, MD, ${ }^{1}$ Roberto L. Flores, MD ${ }^{2}$ \\ ${ }^{1}$ Department of Plastic Surgery, Ochsner Clinic Foundation, New Orleans, LA ${ }^{2}$ Hansjörg Wyss Department of Plastic Surgery, \\ NYU Langone Medical Center, New York, NY
}

Background: In the subunit principle of nasal reconstruction, the valleys and low ridges of the nose are designated as topographic subunits. Surgical scars can be located at the borders of subunits to hide their appearance.

Case Report: A 30-month-old female presented with an obstructing nasal glial heterotopia (nasal glioma). Using the nasal subunit approach, the mass was exposed using an incision along the subunit borders of the nose. The nasal glioma was completely resected, and the internal nasal valve and the deformed lower lateral cartilages were reconstructed through the subunit approach access incision. The final scar was placed along the subunit borders of the nose. At 6-month follow-up, the patient demonstrated no airway obstruction, adequate nasal contour, and an esthetic nasal scar.

Conclusion: The subunit approach for a large, obstructing nasal glial heterotopia allows direct exposure for tumor resection, framework reconstruction, placement of the incision in an esthetic location, and excision of the expanded skin for recontouring of the skin envelope.

Keywords: Glioma, nasal obstruction, surgery-plastic

Address correspondence to Michael T. Friel, MD, Department of Plastic Surgery, Ochsner Clinic Foundation, 1514 Jefferson Hwy., New Orleans, LA 70121. Tel: (504) 842-5998. Email: michael.friel@ochsner.org

\section{INTRODUCTION}

Common midline lesions affecting the pediatric nose include hemangiomas, gliomas, dermoid cysts, and encephaloceles. Although many of these midline masses can be diagnosed preoperatively through computed tomography (CT) and magnetic resonance imaging (MRI), ${ }^{1}$ in some instances, complete excisional biopsy is the only way to reveal the definitive tissue diagnosis. ${ }^{2,3}$ Nasal gliomas, in particular, can be mistaken for other nasal masses, partially because of their relative rarity. Preoperative imaging is obligatory for any midline nasal mass to rule out the possibility of an intracranial communication. Clinicians and pathologists increasingly prefer the term nasal glial heterotopia in lieu of the historic term nasal glioma because the lesions are not neoplasms and have no chance of malignant degeneration. ${ }^{4}$

Burget and Menick ${ }^{5}$ first proposed the subunit principle of nasal reconstruction as an expansion of a reconstructive principle proposed by Gonzalez-Ulloa et al $^{6}$ who designated the entire nose as an esthetic unit of the face. The surface of the nose is crossed by gentle valleys and low ridges that divide it into topographic subunits. The subunits are the dorsum, tip, sidewalls, alar lobules, and soft triangles. ${ }^{5}$ Scars can be located at the borders of subunits to best hide their appearance.

Large nasal gliomas have an associated expansion of the nasal skin envelope that requires tailored resection and re- construction to esthetically redrape the nose after tumor extirpation. Through application of the subunit principle of nasal reconstruction described by Menick and Burget, 5,7 Warren and colleagues described the technique of subunit resection of nasal hemangiomas ${ }^{8}$ that provides excellent exposure and cosmesis. To our knowledge, the subunit approach to a large nasal glial heterotopia has not been previously described. We present the case of a patient with a focal nasal glial heterotopia that was treated using the nasal subunit principle for wide exposure of the tumor and subsequent recontouring of the nasal skin.

\section{CASE REPORT}

A 30-month-old female presented to the vascular anomalies clinic for a soft tissue lesion that completely obstructed the right nasal airway (Figures 1 and 2). Physical examination at this visit revealed a firm, noncompressible mass at the nasal tip, and MRI findings suggested nasal glial heterotopia. The patient underwent resection of the nasal tumor and reconstruction of the upper and lower lateral cartilages through a subunit approach. An incision was made along the subunit borders of the nose between the dorsum and the lateral nasal wall, the ala, and the nasal tip and then along the nasal tip and the soft triangle (Figure 2). This subunit incision provided wide exposure of the nasal mass that grew in a bilobed pattern with a submucosal component 

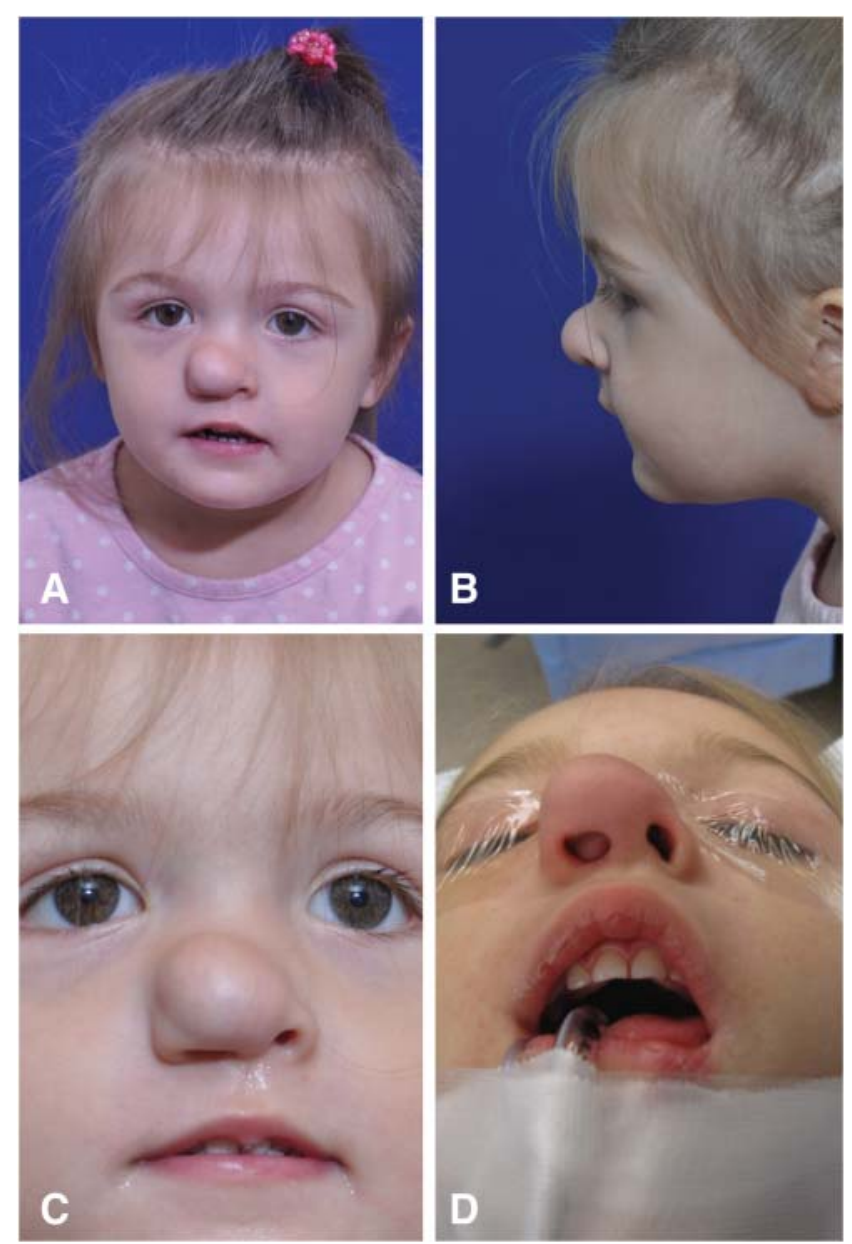

Figure 1. Preoperative anteroposterior (A), lateral (B), midface (C), and worm's eye (D) views of the patient show the large, bulbous mass obstructing the right nostril and distorting the upper lateral and lower lateral cartilages.

inferior to the upper lateral cartilages that obstructed the nasal airway and a subcutaneous component between the upper and lower lateral cartilages and the nasal skin (Figure 3). The $2.5 \times 1.5 \times 2.0-\mathrm{cm}$ mass was carefully excised from the surrounding nasal cartilages, taking advantage of the direct approach offered by the subunit incision. The upper lateral cartilages, the intranasal mucosa, and the lower lateral cartilage on the patient's right side were reconstructed through the subunit incision by direct suturing for repositioning. The skin envelope was then advanced over the defect, and excess skin was trimmed from the medial and lateral flaps to place the final scar along the subunit borders of the nose.

Six months after resection and reconstruction, the patient's nasal scars were esthetic and placed along the subunit borders of the nose (Figure 4). Intranasal examination revealed a well-reconstructed internal nasal valve without evidence of recurrent nasal obstruction.

\section{DISCUSSION}

The subunit approach for resection of nasal glial heterotopia allows for skin resection, wide exposure, and an esthetically pleasing scar. Other surgical options include an open

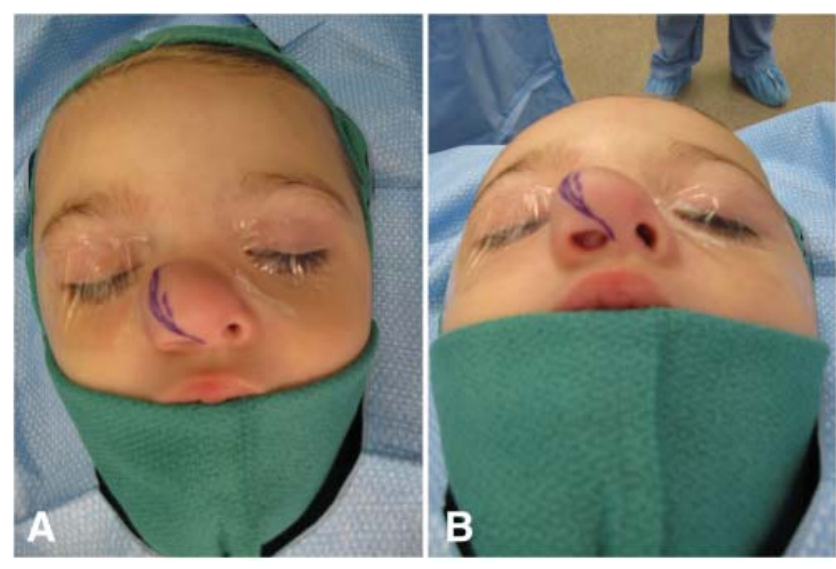

Figure 2. Operative marking and demonstration of nasal obstruction in the anteroposterior (A) and worm's eye (B) views. The marking is placed to allow for the final scar to be located between nasal subunits.

rhinoplasty incision, which has been advocated by McCarthy et al in the treatment of nasal tip hemangiomas. ${ }^{9}$ Although an open rhinoplasty incision produces an esthetic and well-hidden scar, the exposure of the extended area of the lateral and superior aspects of the upper lateral cartilages can be limited. Full exposure can be of particular

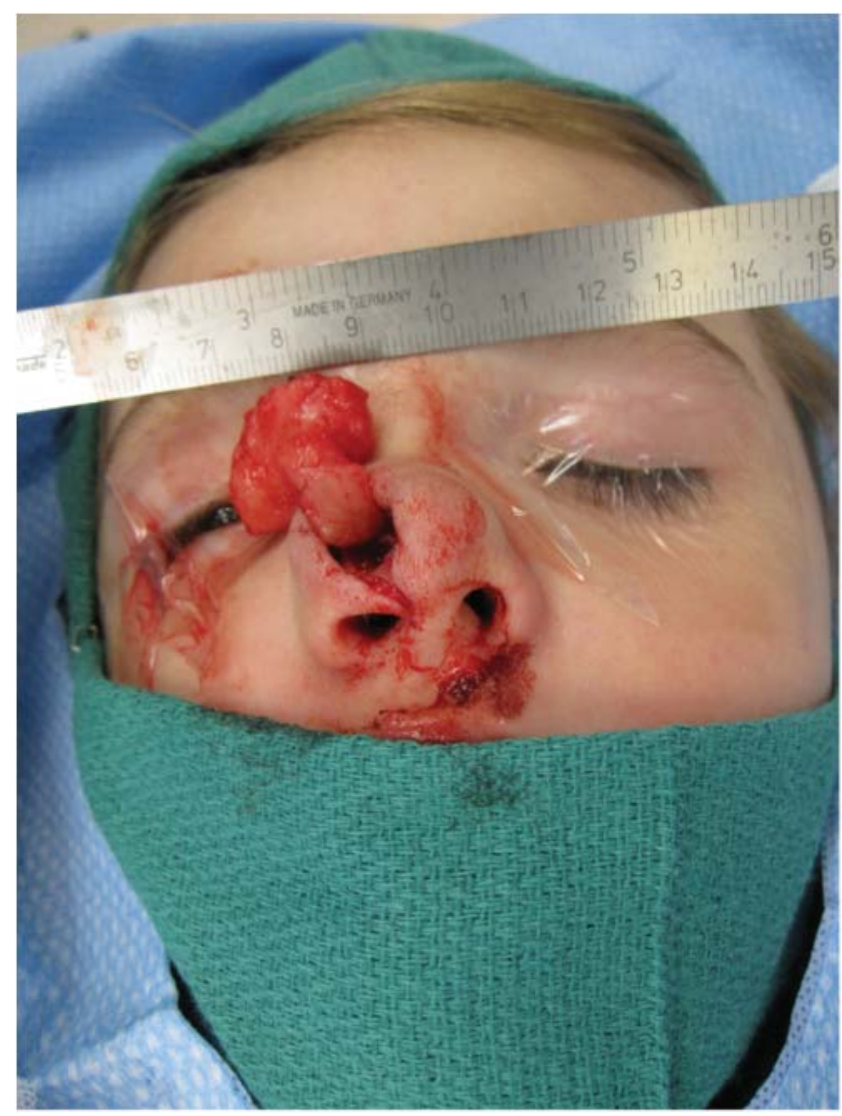

Figure 3. Nasal glial heterotopia dissected prior to excision. The mass measured $2.5 \times 1.5 \times 2.0 \mathrm{~cm}$. 


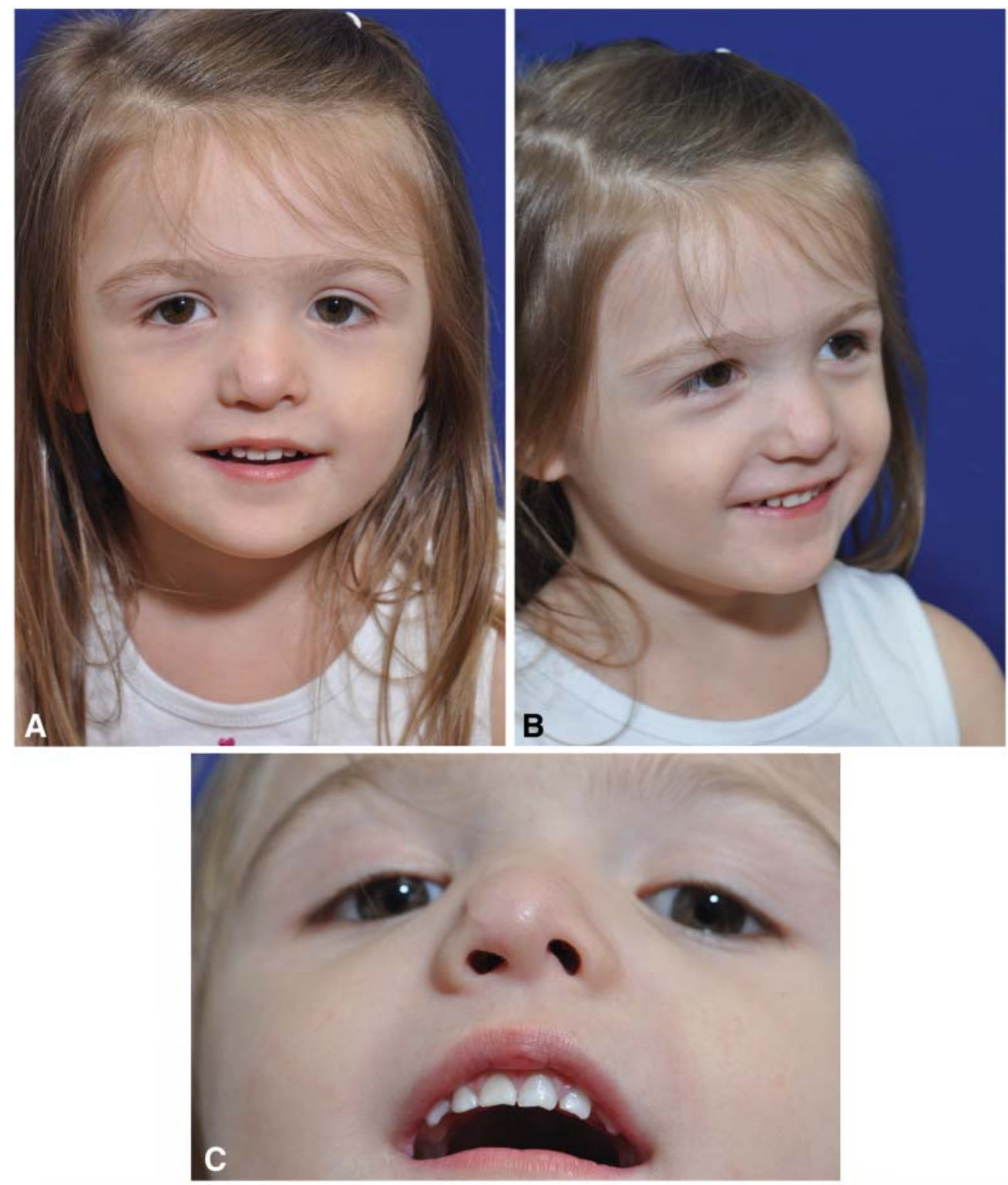

Figure 4. Postoperative anteroposterior (A), oblique (B), and worms-eye (C) views at 6-month follow-up show an esthetically acceptable result with complete resolution of preoperative symptoms.

importance in the excision of large tumors, as was the case in our patient. More important, the use of the open rhinoplasty incision does not address the expanded nasal envelope, leading to fluid accumulation and scar formation at the operative site that ultimately results in recurrence of the deformity. Midline ${ }^{10}$ and L-approach ${ }^{11}$ incisions have also been described; however, lack of adherence to the subunit principles may lead to unesthetic scars.

\section{CONCLUSION}

In our patient, the subunit approach allowed direct exposure of the mass; placement of the incision in an esthetic location; and excision of the expanded, redundant skin for recontouring of the skin envelope. This approach led to a pleasing esthetic result.

\section{ACKNOWLEDGMENTS}

The authors have no financial or proprietary interest in the subject matter of this article.

\section{REFERENCES}

1. Dupin $\mathrm{CL}$, LeJeune FE Jr. Nasal masses in infants and children. South Med J. 1978 Feb;71(2):124-128.

2. Levine MR, Kellis A, Lash R. Nasal glioma masquerading as a capillary hemangioma. Ophthal Plast Reconstr Surg. 1993 Jun;9 (2):132-134.

3. Birnbaum LM, Owsley JQ Jr. Frontonasal tumors of neurogenic origin. Plast Reconstr Surg. 1968 May;41(5):462-470.

4. Penner CR, Thompson LD. Nasal glial heterotopia. Ear Nose Throat J. 2004 Feb;83(2):92-93.

5. Burget GC, Menick FJ. The subunit principle in nasal reconstruction. Plast Reconstr Surg. 1985 Aug;76(2):239-247. 
6. Gonzalez-Ulloa M, Castillo A, Stevens E, Alvarez Fuertes G, Leonelli $F$, Ubaldo F. Preliminary study of the total restoration of the facial skin. Plast Reconstr Surg (1946). 1954 Mar;13(3): 151-161.

7. Burget G. Discussion: reconstruction of the nasal soft triangle subunit. Plast Reconstr Surg. 2013 May;131(5):1051-1054. doi: 10.1097/PRS.0b013e318289ec31.

8. Warren SM, Longaker MT, Zide BM. The subunit approach to nasal tip hemangiomas. Plast Reconstr Surg. 2002 Jan;109(1):25-30.
9. McCarthy JG, Borud LJ, Schreiber JS. Hemangiomas of the nasal tip. Plast Reconstr Surg. 2002 Jan;109(1):31-40.

10. Pitanguy I, Machado BH, Radwanski HN, Amorim NF. Surgical treatment of hemangiomas of the nose. Ann Plast Surg. 1996 Jun;36(6):586-592.

11. van der Meulen JC, Gilbert M, Roddi R. Early excision of nasal hemangiomas: the L-approach. Plast Reconstr Surg. 1994 Sep;94 (3):465-473.

This article meets the Accreditation Council for Graduate Medical Education and the American Board of Medical Specialties Maintenance of Certification competencies for Patient Care and Medical Knowledge. 